\title{
Spiritual Care Needs of Terminal Ill Cancer Patients
}

\author{
Wasinee Wisesrith ${ }^{1}$, Pilaiporn Sukcharoen ${ }^{2 *}$, Kanittha Sripinkaew ${ }^{3}$
}

\begin{abstract}
Objective: Terminal ill cancer patients experience spiritual distress and need spiritual care. They are in need of palliative care in the long term to alleviate suffering. The aim of the study was to investigate spiritual needs of terminal ill cancer patients. Methods: This study is a cross-sectional study. The participants were Thai terminal ill cancer patients from seven hospitals in northern, northeast, central, and southern regions of Thailand. Three-hundred-and-twenty-two participants were purposively selected. The instrument used in this study was a spiritual needs scale, consisting of 41 items with 4-point rating scale. Statistical analysis was F-test for one-way analysis of variance. Results: The majority of terminal ill cancer patients aged fifty-one years old. Most of them were females with end stage cancer status that did not respond to treatment. The overall spiritual needs of terminal ill cancer patients were at the moderate level $(\mathrm{M}=$ 18.21, $\mathrm{SD}=2.56$ ). The highest mean was found in the 'prepare for death' dimension, followed by the 'have meaning, values, and life purposes' and the 'have opportunity to pursue most important things in life' dimensions respectively and had different spiritual needs among status $(\mathrm{F}(3,318)=3.66, \mathrm{p}<0.05)$, number of family members $(\mathrm{F}(8,313)=5.07$, $\mathrm{p}<0.05)$, living with family $(\mathrm{F}(2,319)=3.91, \mathrm{p}<0.05)$, and spiritual anchor $(\mathrm{F}(2,319)=4.13, \mathrm{p}<0.05)$. Conclusion: The study results provide insight into spiritual needs of terminal ill cancer patients which assists in improving the provision of holistic care to let the patients be happy at end-of-life phase.
\end{abstract}

Keywords: Palliative care- spiritual care- cancer patients

Asian Pac J Cancer Prev, 22 (12), 3773-3779

\section{Introduction}

Currently, worldwide economic, social, and health conditions change rapidly. Also, there is a problem related to important health system in Thailand. There is continuing growth in the number of older adults, patients with chronic diseases, and cancer patients. Based on the National Cancer Institute of Thailand, 130,000 new cancer cases are found yearly. $39.96 \%$ of these cases were people aged 60 and over. In addition, cancer was the number one cause of death: there were $107.9 \%, 113.7 \%$, and $119.3 \%$ per 100,000 persons in 2014, 2015, and 2016 consecutively (Health Administration Division, 2012).

Moreover, patients are generally diagnosed as having incurable disease if cancer develops to the advanced stage. That affects the patients in all aspects of their lives, including physical, mental, psychosocial, and spiritual dimensions. Accordingly, terminal ill cancer patients are in need of palliative care in the long term to alleviate suffering resulted from the disease and burden of care the family carry (Daaleman et al., 2014; Arisanti et al., 2019). Health care professionals should have knowledge of patient care processes. Meanwhile, they should have knowledge of how to provide care for terminal ill cancer patients to alleviate the patients' suffering as well as to promote better quality of life (Gillan et al., 2014; Ozveren and Kirca, 2019; Sukcharoen et al., 2020).

Palliative care is an important care provided for terminal ill cancer patients. It is the science of holistic care for terminal ill patients and their families which focuses on physical, mental, social, and spiritual care (Paganini and Bousso, 2015; Mishra et al., 2020). In addition, it involves knowledge of holistic care that is built on an integration between healthcare providers, community, and public policy, aiming to alleviate suffering and promote quality of life of patients and families (Wisesrith et al., 2019). According to the aforementioned information, it is important that healthcare professionals, specifically registered nurses become aware of the importance of providing holistic care emphasizing spiritual care to terminal ill cancer patients. That is to serve care needs in all areas as well as to help the patients spend the rest of their lives with peace (Gillan et al., 2014; Arisanti et al., 2019; Kong and Guan, 2019).

Based on a review of spirituality concepts, spirituality of terminal ill cancer patients was found to vary, depending on perceptions, attitudes, experiences, and sociocultural conditions (Mishra et al., 2020; Sukcharoen et al , 2020); especially when the patients were facing suffering and incurable diseases. Subsequently, the patients experienced 
spiritual distress and needed spiritual care. If any terminal ill cancer patient developed the perception and achieve good spirituality, they would have positive experience of the illness and be ready to face with terminal ill conditions. On the contrary, if the patient had negative experience of the illness, they would suffer more and not be ready to face end-of-life crises (Daaleman et al., 2014; Arisanti et al., 2019; Wisesrith et al., 2019; Kong and Guan, 2019). Therefore, it is necessary that registered nurses have understanding about the spiritual needs of terminal ill cancer patients and families to provide efficient palliative care.

However, there is lack of specific health care systems for terminal patients in the context of the provision of care for terminal ill cancer patients in Thai hospitals. There is no agency mainly in charge of palliative care provision, particularly in the dimensions of mental and spirituality of patients. The overall picture of care in Thailand is the provision of care in general, primarily focus on the physical dimension (Wisesrith et al., 2019; Sukcharoen et al., 2020; Sukcharoen et al., 2020). Moreover, previous studies mainly focus on the structure of palliative care related to terminal patients, palliative care nursing (Giovanni, 2012; Beccaro et al., 2013; Benito et al., 2014), and the development of tools to measure and evaluate quality of care for terminal patients (Daaleman et al., 2014; Wisesrith et al., 2019). The study on spiritual needs of terminal ill cancer patients in Thailand and other countries has not been found. Accordingly, terminal ill cancer patients do not receive sufficient and efficient spiritual care (Sukcharoen et al., 2020).

This study primarily applies a concept of palliative care to encourage patients to accept the nature of life and death (Wannapornsiri, 2018; Wisesrith et al., 2019; Sukcharoen et al., 2020; Sukcharoen et al., 2020). The study also includes the concept of spirituality and spiritual care, paying attention to and giving respect for personal perception of patients as well as encouraging terminal ill cancer patients and families to face and adapt to suffering at end-of-life phase (Wisesrith et al., 2019; Sukcharoen et al., 2020). That is to gain better understanding about spiritual needs of the patients and families. Understanding of spiritual needs of terminal ill cancer patients is the fundamental information that nurses and multidisciplinary teams providing palliative care and end-of-life care can be applied to provide holistic care for terminal ill cancer patients, including physical, mental, social, and spiritual dimensions. That is to alleviate the patients' pain and suffering as well as to develop an approach to provide spiritual care for terminal ill cancer patients efficiently.

According to the aforementioned reasons, the researcher aims to study spiritual needs of terminal ill cancer patients in the context of Thai culture to gain insight about their spiritual needs which assists in improving the provision of holistic care to let the patients be happy at end-of-life phase.

\section{Materials and Methods}

This study is a cross-sectional study Participants
The participants were purposively selected based on the following criteria: terminal ill cancer patients who did not respond to the treatment, both male and female, admitted in the hospital, aged 40 and over, had the performance score of $40-70 \%$, had full consciousness, could communicate normally, and were willing to participate in this study. The sample size was determined applying rule of thumb: Number of multivariable was 1020 times greater than the number of observable variables. Also, the research team added $15 \%$ of the sample size to increase the reliability; subsequently the sample size of 322 was derived.

\section{Research instruments}

The research instrument was the spiritual needs scale. It was developed applying a systematic literature review integrated with in-depth interview with terminal patients, family caregivers, and registered nurses with experience in providing care for patients. Content validity was validated by 17 experts, a Delphi technique was performed. The obtained median was 4.84 and interquartile range was 0.82. For reliability testing, the Cronbach's alpha of 0.89 was found (Wisesrith et al., 2019).

The research instrument was built on Wisesrith et al.'s study investigating the development and preliminary testing of the spiritual care need assessment scale for terminal patients in Thai cultural context. It was found that spirituality of terminal ill cancer patients was varied depending on perceptions, attitudes, experiences, and sociocultural conditions (Wisesrith et al., 2019) and was rooted in religious beliefs and values in life (Sukcharoen et al., 2020; Sukcharoen et al., 2020).

The spiritual needs scale consisted of two sections. Section 1 Personal information, there were nine multiplechoice questions asking about age, marital status, number of family members, living with family, types of accommodation, religious belief, frequency of prayer, frequency of religious practice, and spiritual anchor. Section 2 Spiritual needs scale, it was a 41 item with 4-point rating scale and consisted of eight dimensions. There were six items in the dimension 'have meaning, values, and life purposes', three items in the dimension 'gain love and encouragement from families and close persons', four items in the dimension 'review of life experience in the past, six items in the dimension 'have opportunity to pursue most important things in life', eight items in the dimension 'prepare for death', six items in the dimension 'have opportunity to practice activities related to beliefs and faith as well as perform religious activities/rituals', four items in the dimension 'live in tranquil environment and atmosphere', and four items in the dimension 'needs to receive assistance in protecting human dignity' (Wisesrith et al., 2019).

\section{Data collection}

Letters of request for data collecting issued by the researcher were sent to the directors of seven hospitals in northern, northeast, central, and southern regions of Thailand. The attached documents consisted of one copy of a questionnaire, one copy of a research proposal, and one copy of a certificate of approval issued by the 
Institutional Review Board. The selected hospital met the following criteria: a hospital that implemented palliative care system with palliative care nurses.

After getting approval for data collecting, the research team contacted head nurses and held a meeting with research assistants to clarify the objectives of the study and the measurement procedures. The research assistants were those with the following qualifications: hospital staff who were in charge of palliative care provision, had experience in the care, and had been trained for palliative care. Afterwards, questionnaires, consent forms, and participant information sheets were sent to the research assistants by post. The questionnaires were sent to measure spiritual needs of the participants; subsequently, the completed ones were sent back to the research team upon the due date.

\section{Data analysis}

(a) Personal information of terminal ill cancer patients was analyzed applying frequency and percentage.

(b) Levels of spiritual needs of terminal ill cancer patients, both overall and by dimension, were analyzed applying mean and standard deviation. Interpreting spiritual need scale used mean scale for the categorized level of each dimension with 3 levels were derived: low level (1-10 score), medium level (11-20 score), and high level (21-30 score) (Wisesrith et al., 2019).

(c) Spiritual needs of terminal ill cancer patients with varied age, marital status, number of family members, living with family, type of accommodation, religious belief, frequency of prayer, frequency of religious practice, and spiritual anchor were compared applying an F-test for one-way analysis of variance. Pair comparison was performed when any mean difference was found.

\section{Results}

\section{Personal information}

Personal information of 322 terminal ill cancer patients: The majority of terminal ill cancer patients aged between 51 and 60 and between 61 and $70(27.95 \%$ and $27.95 \%$ respectively). For marital status, the majority of patients had married status $(68 \%)$. For number of family members, most patients had four family members $(28 \%)$.
For living with family, the participants mostly lived with families (86.3\%). Type of accommodation mostly reported was stand-alone house (87.9\%). For religion, Buddhism (96\%) was most practiced. Praying daily $(28.6 \%)$ was most reported. For frequency of religious practice, perform sometimes $(46.9 \%)$ was mostly reported. For spiritual anchor, having spiritual anchor $(68.9 \%)$ was mostly reported.

\section{The Level of spiritual needs of terminal ill cancer patients}

The overall spiritual needs of terminal ill cancer patients were at the moderate level $(\mathrm{M}=18.21, \mathrm{SD}=2.56)$. The dimension with the highest mean score was 'prepare for death' which was at the high level $(\mathrm{M}=28.85$, SD $=3.49$ ). That was followed by two dimensions: 'have meaning, values, and life purposes' which was at the high level $(\mathrm{M}=21.86, \mathrm{SD}=2.59)$ and 'have opportunity to pursue most important things in life' which was also at the high level $(\mathrm{M}=21.40, \mathrm{SD}=2.83)$. The dimension with the lowest mean score was 'live in tranquil environment and atmosphere' which was at the moderate level $(\mathrm{M}=$ $14.67, \mathrm{SD}=1.81)$. These results are as shown in Table 1 .

The one-way analysis of variance to compare means of spiritual needs of terminal ill cancer patients

Terminal ill cancer patients had different spiritual needs at the statistical significance level of .05 among status $(\mathrm{F}(3,318)=3.66, \mathrm{p}<0.05)$, number of family members $(F(8,313)=5.07, p<0.05)$, living with family $(\mathrm{F}(2,319)=3.91, \mathrm{p}<0.05)$, and spiritual anchor $(\mathrm{F}(2,319)$ $=4.13, \mathrm{p}<0.05)$. These results are as shown in Table 2 .

To identify the difference in spiritual needs of terminal ill cancer patients with varied marital status, number of family members, living with family, and spiritual anchor; the Scheffe post hoc comparison was performed. Results revealed that the spiritual needs were higher for the terminal ill cancer patients with married status $(\mathrm{M}=147.58)$ than those with widowed status ( $M$ $=142.01)$; were higher for the patients with 3-4 family members $(\mathrm{M}=150.69)$ than those with $1-2$ family members $(\mathrm{M}=140.79)$ and those with 5 members and over $(M=142.14)$; were higher for the patients who lived with family $(\mathrm{M}=146.77)$ than those who lived alone $(\mathrm{M}=139.48)$; and were higher for the patients with one spiritual anchor $(\mathrm{M}=147.84)$ than those who had none

Table 1. The Mean and Level of Overall and Each Dimension of Spiritual Needs of Terminal Ill Cancer Participants $(\mathrm{N}=322)$

\begin{tabular}{lcc}
\hline Spiritual needs of terminal ill cancer participants & Mean \pm SD & Level \\
\hline 1. have meaning, values, and life purposes & $21.86 \pm 2.59$ & High \\
2. gain love and encouragement from families and close persons & $11.11 \pm 1.40$ & Medium \\
3. review of life experience in the past & $12.43 \pm 2.66$ & Medium \\
4. have opportunity to pursue most important things in life & $21.40 \pm 2.83$ & High \\
5. prepare for death & $28.85 \pm 3.49$ & High \\
6. have opportunity to practice activities related to beliefs and faith was well as perform religious & $20.66 \pm 3.73$ & High \\
activities/rituals & $14.67 \pm 1.81$ & Medium \\
7. live in tranquil environment and atmosphere & $14.76 \pm 1.97$ & Medium \\
8. needs to receive assistance in protecting human dignity & $18.21 \pm 2.56$ & Medium \\
Overall &
\end{tabular}


Table 2. The Results for One-Way Analysis of Variance to Compare Means of Spiritual Needs of Terminal Ill Cancer Patients by ANOVA

\begin{tabular}{|c|c|c|c|c|c|c|}
\hline Factor & & $S S$ & $d f$ & $M S$ & $F$ & $p$ \\
\hline \multirow[t]{2}{*}{ Age } & Between group & 12393.52 & 51 & 243.01 & 1.09 & 0.32 \\
\hline & Within group & 60117.26 & 270 & 222.66 & & \\
\hline \multirow[t]{2}{*}{ Marital status } & Between group & 2422.55 & 3 & 807.52 & 3.66 & $0.01^{*}$ \\
\hline & Within group & 70088.24 & 318 & 220.4 & & \\
\hline \multirow{2}{*}{$\begin{array}{l}\text { Number of family } \\
\text { members }\end{array}$} & Between group & 8313.01 & 8 & 1039.13 & 5.07 & $0.00 *$ \\
\hline & Within group & 64197.77 & 313 & 205.1 & & \\
\hline \multirow[t]{2}{*}{ Living with family } & Between group & 1735.83 & 2 & 867.92 & 3.91 & $0.02 *$ \\
\hline & Within group & 70774.95 & 319 & 221.87 & & \\
\hline \multirow[t]{2}{*}{ Type of accommodation } & Between group & 1088.92 & 6 & 181.49 & 0.8 & 0.57 \\
\hline & Within group & 71421.87 & 315 & 226.74 & & \\
\hline \multirow[t]{2}{*}{ Religious belief } & Between group & 298.75 & 3 & 99.58 & 0.44 & 0.73 \\
\hline & Within group & 72212.03 & 318 & 227.08 & & \\
\hline \multirow[t]{2}{*}{ Frequency of prayer } & Between group & 1492.54 & 4 & 373.14 & 1.67 & 0.16 \\
\hline & Within group & 71018.24 & 317 & 224.03 & & \\
\hline \multirow{2}{*}{$\begin{array}{l}\text { Frequency of religious } \\
\text { practice }\end{array}$} & Between group & 1514.6 & 4 & 378.65 & 1.69 & 0.15 \\
\hline & Within group & 70996.18 & 317 & 223.96 & & \\
\hline \multirow[t]{2}{*}{ Spiritual anchor } & Between group & 1828.68 & 2 & 914.34 & 4.13 & $0.02 *$ \\
\hline & Within group & 70682.1 & 319 & 221.57 & & \\
\hline
\end{tabular}

Table 3. Mean Comparisons of Spiritual Needs of Terminal Ill Cancer Participants with Marital Status by the Scheffe Post Hoc Comparison

\begin{tabular}{lcccc}
\hline & & Single & Married & Widowed \\
\hline Marital status & M & 141.64 & 147.58 & 142.01 \\
Single & 141.64 & & -5.93 & -0.36 \\
Married & 147.58 & 5.93 & & $5.57^{*}$ \\
Widowed & 142.01 & 0.36 & $-5.57^{*}$ & \\
\hline${ }^{*} p 0.05$ & &
\end{tabular}

$(M=140.88)$. The results are as shown in Tables 3-6.

\section{Discussion}

The result of this study showed that the overall mean of spiritual needs of terminal ill cancer patients was at the moderate level. There are some possible explanations for such result. It might be the fact that these patients depend on medical devices to live because the diseases are incurable. Therefore, they endure physical, mental, social, and spiritual distress (Wisesrith et al., 2019; Sukcharoen et al., 2020). Moreover, due to the fact that terminal ill cancer patients are unable to communicate their needs, their physical conditions are mainly cared for; while other dimensions of their needs are neglected (Sukcharoen et al., 2020). Registered nurses are the health care providers playing an important role in the delivery of holistic care

Table 4. Mean Comparisons of Spiritual Needs of Terminal Ill Cancer Participants with Number of Family Members by the Scheffe Post Hoc Comparison

\begin{tabular}{lcccc}
\hline & & $1-2$ members & $3-4$ members & 5 members and over \\
\hline Number of family members & $\mathrm{M}$ & 140.79 & 150.69 & 142.14 \\
$1-2$ members & 140.79 & & $-9.90^{*}$ & -1.35 \\
$3-4$ members & 150.69 & $9.90^{*}$ & & $8.55^{*}$ \\
5 members and over & 142.14 & 1.35 & $-8.55^{*}$ & \\
${ }^{*} p<0.05$ & &
\end{tabular}

Table 5. Mean Comparisons of Spiritual Needs of Terminal Ill Cancer Participants with Living with Family by the Scheffe Post Hoc Comparison

\begin{tabular}{lcccc}
\hline & & Live alone & Live with family & Live with friends \\
\hline Living with family & $\mathrm{M}$ & 139.48 & 146.77 & 139.67 \\
Live alone & 139.48 & & $-7.28^{*}$ & -0.18 \\
Live with family & 146.77 & $7.28^{*}$ & & 7.1 \\
Live with friends & 139.67 & 0.18 & -7.1 & \\
\hline${ }^{*} p 0.05$ & & &
\end{tabular}


Table 6. Mean Comparisons of Spiritual Needs of Terminal Ill Cancer Participants with Spiritual Anchor by the Scheffe Post Hoc Comparison

\begin{tabular}{lcccc}
\hline & & None & 1 spiritual anchor & 2 spiritual anchor and over \\
\hline Spiritual anchor & $\mathrm{M}$ & 140.88 & 147.84 & 146.33 \\
None & 140.88 & & $-6.95^{*}$ & -5.45 \\
1 spiritual anchor & 147.84 & $6.95^{*}$ & & 1.50 \\
2 spiritual anchor and over & 146.33 & 5.45 & -1.50 & \\
$*^{*} p 0.05$ & & &
\end{tabular}

for terminal ill cancer patients to alleviate suffering for the patients and their families (Gillan et al., 2014; Arisanti et al., 2019; Ozveren and Kirca, 2019; Mishra et al., 2020).

Considering overall picture of the study results, spiritual needs of terminal ill cancer patients in the dimension of 'prepare for death' had the highest mean score, followed by 'have meaning, values, and life purposes' and 'have opportunity to pursue most important things in life'. It can be explained that terminal ill cancer patients need to accept the illness they are facing, to learn to take care of themselves in line with their own religious beliefs and spiritual anchors, and to have sufficient time to reflect on nature of death -i.e., it is common for everyone (Astrow, 2012; Kong and Guan, 2019). Lin et al., (2015) provided supporting argument stating that terminal ill cancer patients viewed life as more meaningful and have peace of mind if they had religious beliefs and faith, positive attitudes towards life, and love from family.

In addition, Vilalta et al., Mishra et al., and Masso et al. explain that if end-of-life needs of terminal ill cancer patients are fulfilled and they feel that hope exists, their quality of life would get better. Also, they will leave this world in peace. Accordingly, registered nurses should provide preparation for end-of-life phase for terminal ill cancer patients and their families. That is to enable the patients to understand the illness, accept the conditions of the diseases, and accept death (Health Administration Division, 2012; Wannapornsiri, 2018; Sukcharoen et al., 2020). The patients should receive holistic care, especially spiritual care to leave this world in peace with human dignity.

Additionally, spiritual care provided for terminal ill cancer patients is important. It is a challenging issue for registered nurses and health care professionals regarding how to serve the patients spiritual needs, alleviate the suffering, let them be happy at end-of-life phase, and let them have better quality of life (Wannapornsiri, 2018; Wisesrith et al., 2019). Thus, spiritual care is an important approach that all registered nurses should keep in mind and provide it for terminal ill cancer patients. That is to allow the patients to have good care and get their spiritual needs fulfilled efficiently, eventually the patients die with human dignity (Leow et al., 2014; Mishra et al., 2020).

The result of this study also showed that terminal ill cancer patients with varied marital status, number of family members, living with family, and spiritual anchor differed in spiritual needs at the statistical significance level of .05. A possible explanation for this result might be the conditions of incurable diseases, terminal ill cancer patients experience physical, mental, social, and spiritual distress. Their spiritual needs are varied in relation to beliefs, customs, tradition and culture, and religions and spiritual anchor that the patients and families have practiced (Wisesrith et al., 2019; Sukcharoen et al., 2020). The provision of holistic care for terminal ill cancer patients, especially the care related to the dimension of spirituality and beliefs of the patients and families is valuable and important in the area of palliative care for terminal ill cancer patients. It aims to let the patient be happy at end-of-life phase and live this world with human dignity (Daaleman et al., 2014; Kong and Guan, 2019).

Considering the study results: (a) terminal ill cancer patients with married status had higher mean score of spiritual needs than those with widowed status. (b) terminal ill cancer patients with 3-4 family members had higher mean score of spiritual needs than those with 1-2 family members and those with 5 family members and over. (c) Terminal ill cancer patients who lived with family had higher mean score of spiritual needs than those who lived alone. It can be explained that terminal ill cancer patients feel happy when perceiving that they are valuable to others and families as well as able to perform behaviors that benefit others (O'Brien, 2017). These patients are happy about being useful for others. They perceive life as valuable, have self-esteem, satisfy with current situation in life, and have better spiritual well-being (Wisesrith et al., 2019), resulting in having higher spiritual needs than terminal ill cancer patients with widowed status or lived alone.

In addition, (d) Terminal ill cancer patients with one spiritual anchor had higher mean score of spiritual needs than those who had none. It can be explained that terminal ill cancer patients who have faith in religions will perform behaviors in line with the principles of their religions, they will have faith in rules, custom, tradition, values or culture of the society/ sacred objects (Sukcharoen et al., 2020). This will lead to living together in peace, finding happiness in life, and having better spiritual well-being (O'Brien, 2017; Wisesrith et al., 2019), resulting in having higher spiritual needs than terminal ill cancer patients who had no spiritual anchor.

Moreover, spiritual needs of terminal ill cancer patients are varied depending on experience, culture, tradition, religion, and values. Having incurable disease is a major factor causing suffering in all areas, especially mental and spiritual distress. Some cases may be unable to accept death and end-of-life phase they are encountering, resulting in spiritual distress and no inner calm (Wisesrith et al., 2019). On the contrary, if terminal ill cancer patients improve perception and achieve good spirituality; they 
will have positive experience related to their current illness, resulting in better quality of life (Boonyarat and Uppanisakorn, 2014). Spiritual care responsive to terminal ill cancer patients' beliefs, faith, and religious rituals is one component of efficient holistic care.

In summary, religious beliefs and faith directly influence quality of life of terminal ill cancer patients. Each individual patient has different religious beliefs and needs (Shim et al., 2011). When these needs are fulfilled, the patients will have peaceful life as well as will be able to accept illness' conditions and death better. Registered nurses and health care providers should assist terminal ill patients and their families in performing religious rituals in line with the patients' own culture and beliefs (Boonyarat and Uppanisakorn, 2014) to serve their spiritual needs and promote quality of life of terminal ill cancer patients and their families (Lin et al., 2015).

In conclusion, terminal ill cancer patients are in need of palliative care in the long term to alleviate suffering resulted from the disease and burden of care the family carry. Health care professionals should have knowledge of patient care process and how to provide care for terminal ill cancer patients to alleviate the patients' suffering, specifically spiritual care. That is to serve care needs in all areas as well as to help the patients spend the rest of their lives with peace. The spirituality of terminal ill cancer patients varied depending on perceptions, attitudes, experiences, and sociocultural conditions, especially when the patients facing suffering and incurable diseases. The patients have spiritual distress and need spiritual care. If any terminal ill cancer patient improve perception and achieve good spirituality, they will have positive experience of the illness and be ready to face with terminal ill conditions. Health care professionals and registered nurses should be encouraged to continually assess spiritual needs of terminal ill cancer patients to accurately assess spiritual needs of each individual patient and provide specific care accordingly.

\section{Limitations of the study}

This study used data obtained only from Thai terminal ill cancer patients who did not respond to treatment from seven hospitals in northern, northeast, central, and southern regions of Thailand. Therefore, the results may not be applicable to Thai terminal ill cancer patients who respond well to treatment.

\section{Implications for nursing practice}

The study results provide insight into spiritual needs of terminal ill cancer patients and their families, which assists in improving the provision of holistic care to let the patients be happy at end-of-life phase. To alleviate the patients' suffering and improve their quality of life, health care professionals and registered nurses should be provided with knowledge about the assessment of spiritual needs of terminal ill cancer patients to accurately assess spiritual needs of each individual patient. Also, they should be encouraged to continually assess spiritual needs of terminal ill cancer patients to accurately assess spiritual needs of each individual patient and provide specific care accordingly.

\section{Author Contribution Statement}

Wasinee Wisesrith, Pilaiporn Sukcharoen, Kanittha Sripinkaew performed acquisition of patient data, analysis and interpretation of patient data. Wasinee Wisesrith, Pilaiporn Sukcharoen developed the study conception and design. Pilaiporn Sukcharoen performed critical revision of the manuscript. All authors have read and agreed to the final version of this manuscript.

\section{Acknowledgements}

This study is part of a research project funded by the National Research Council of Thailand in the fiscal year of 2017. The authors would like to express their gratitude to Chulalongkorn University for their approval and assistance of this study. The study was approved by the Institutional Review Board of Chulalongkorn University (CU 187.1/59). Informed consent was obtained from all participants. The authors declare no conflict of interest.

\section{Funding Statement}

This research is part of a research project funded by the National Research Council of Thailand in the fiscal year of 2017 .

\section{References}

Arisanti N, Sasongko E, Pandia V, et al (2019). Implementation of palliative care for patients with terminal diseases from the viewpoint of healthcare personnel. BMC Res Notes, 12, 1-5.

Astrow BA (2012). A Chinese version of the spiritual needs assessment for patients survey instrument. J Palliat Med, 15, 1297-305.

Beccaro M, Aprile LP, Scaccabarozzi G, et al (2013). Survey of Italian general practitioners knowledge, opinions, and activities of palliative care. J Pain Symptom Manage, 46, 335-44.

Benito E, Oliver A, Galiana L, et al (2014). Development and validation of a new tool for the assessment and spiritual care of palliative care patients. J Pain Symptom Manage, 47,1008-18.

Boonyarat J, Uppanisakorn S (2014). The spirituality care in intensive patients and familys in intensive care unit: Nursing Experiences. Princess Narathiwat Univ J, 4, 1-13.

Daaleman PT, Reed D, Cohen W, et al (2014). Development and preliminary testing of the quality of spiritual care scale. $J$ Pain Symptom Manage, 47, 793-800.

Gillan CP, Van DP, Jeong S (2014). End of life care education, past and present: A review of the literature. Nurse Educ Today, 34, 331-42.

Giovanni L (2012). End of life care in the United States: Current Reality and Future Promise A Policy Review. Nurs Econ, 30, 127-34.

Health Administration Division (2012). Establishing a health service system development plan [Online]. Available: http:// bps.ops.moph.go.th. [Accessed 27 March 2020].

Kong S, Guan N (2019). Burden in family caregivers of cancer patients: The Association with Depression, Religiosity and Religious Coping. Asian Pac J Cancer Care, 4, 171-82.

Leow M, Chan M, Chan S (2014). Predictors of change in quality of life of family caregivers of patients near the end of life with advanced cancer. Cancer Nurs, 37, 391-9.

Lin YL, Rau KM, Liu YH, et al (2015). Development and 
validation of the Chinese Version of Spiritual Interests Related Illness Tool for patients with cancer in Taiwan. Eur J Oncol Nurs, 19, 589-94.

Masso M, Allingham S, Johnson E, et al (2016). Palliative care problem severity score: Reliability and acceptability in a national study. Palliat Med, 30, 479-85.

Mishra S, Gupta R, Bharati S, et al (2020). Transmutation of spiritual credence during COVID-19 Era in cancer patients: A Case Series. Asian Pac J Cancer Care, 5, 129-32.

O'Brien ME (2017). Spirituality in nursing: Standing on holy ground. Jones \& Bartlett Learning.

Ozveren H, Kirca K (2018). Influence of palliative care training on last-year nursing department students' perception on regarding spirituality and spiritual care: A Single group pretest-posttest intervention study. J Relig Health, 58, 860-9.

Paganini CM, Bousso SR (2015). Nurses' autonomy in end-of--life situations in intensive care units. Nurs Ethics, 22, 803-14.

Shim E, Lee KS, Park J H, et al (2011). Comprehensive needs assessment tool in cancer (CNAT): the development and validation. Support Care Cancer, 19, 1957-68.

Sukcharoen P, Sakunpong N, Sripa K (2020). Spiritual dimension in palliative care from the perspective of Thai palliative caregivers. Int J Palliat Nurs, 26, 70-4.

Sukcharoen P, Sakunpong N, Sripa K (2020). Effectiveness of transformative learning on spirituality in palliative care among nursing students: A Mixed Methods Study. J Behav Sci, 15, 19-33.

Vilalta A, Valls J, Porta J, et al (2014). Evaluation of spiritual needs of patients with advanced cancer in a palliative care unit. $J$ Palliat Med, 17, 592-9.

Wannapornsiri C (2018). The experiences of family caregivers providing palliative cancer care in Thailand. Int $J$ Palliat Nurs, 24, 559-65.

Wisesrith W, Soonthornchaiya R, Sukcharoen P (2019). Development and preliminary testing of the spiritual care need assessment scale for end of life patients in Thai cultural context. $B C N, \mathbf{3 5}, 163-74$.

\section{c) (1) (8)}

This work is licensed under a Creative Commons AttributionNon Commercial 4.0 International License. 